\title{
An Objective Train Timetabling Quality Evaluation Method
}

\author{
Feng Jiang, Da-ben Yu, and Shao-quan Ni \\ School of Transportation and Logistics, National Railway Train Diagram Research and Training Center, \\ National and Local Joint Engineering Laboratory of Comprehensive Intelligent Transportation, 111 North 1 Section, \\ Beierhuan Road, Jinniu District, Chengdu 610031, China \\ Correspondence should be addressed to Shao-quan Ni; nishaoquan@126.com
}

Received 8 October 2016; Revised 14 December 2016; Accepted 9 May 2017; Published 5 June 2017

Academic Editor: Andrea L. Facci

Copyright (C) 2017 Feng Jiang et al. This is an open access article distributed under the Creative Commons Attribution License, which permits unrestricted use, distribution, and reproduction in any medium, provided the original work is properly cited.

\begin{abstract}
The train timetable dominates the rail traffic organization. The timetabling quality should be evaluated to check the work skill of train timetable managers. The values of existing timetable evaluation indexes vary with infrastructure features and traffic flow; therefore, they are not comparable in fact. Furthermore, subjective inputs like expert scores are involved in evaluation; this will lead to unreliable results because the experts may have different opinions. To overcome these shortages, we propose a relative train path efficiency index by taking the train paths as production units. Each unit consumes some transport resources and produces some feedback outputs. A DEA model is applied to compute the train path efficiency. Two statistical functions of train path efficiency are used to evaluate the timetabling quality. We verify our method with real-world timetables. First, we use the Shibantan-to-Xinqiao line timetable to test the relative feature of the index proposed, and the results show that the train path efficiency value is relative and can reflect whether the stops are evenly distributed or not. Second, we evaluate the timetabling quality of another two timetables of the Qingdao-to-Jinan line with different traffic flows, and the results show that, compared with the 2012 timetable, the timetabling quality decreased in 2013.
\end{abstract}

\section{Introduction}

A train timetable defines the departure and arrival times of a train at each station. The Train Timetabling Problem (TTP) is mainly about setting a feasible path for each train by displaying and adjusting the arrival and departure times of the train (see, e.g., Caprara et al. [1]). From this point of view, the evaluation of the train timetabling quality involves evaluating the TTP process mentioned above, particularly the distribution and adjustment of the train paths.

A timetable can be made by a computer or manually. For cases of simple traffic, computer timetabling can provide a good solution, while for large-scale, complex traffic, it is difficult to generate a timetable completely automatically. Therefore, manual adjustments are necessary. In China, where the timetable is nonperiodic and the scale of the rail network is large, at the timetabling stage, a lot of readjustments are required due to the complexity of the problem, so we employ a manual timetabling process. The person in charge of this job is usually known as the timetable manager (TM). As the TTP is NP-hard [1], it is difficult to find the optimal solution for all train paths; thus, different TMs can reach different solutions, and the work skill of the TM will eventually affect the timetable quality. Usually, there are several TMs working on timetabling, each of whom is in charge of different lines. Nowadays, we are facing the problem of how to evaluate the work skill of TMs, in other words, how to find a way to evaluate the train timetabling quality (TTQ).

The TTP is recognized as one of the most important problems in rail traffic organization; a lot of research focuses on this field. In Caprara et al.s study [1], a single one-way track TTP is studied and an ILP model is used, where the ILP is relaxed in a Lagrangian way and a heuristic method is used to schedule each train while keeping it as close as possible to the ideal timetable. In Brännlund et al.s study [2], a single track, two-way line case is studied and the Lagrangian relaxation method is used to solve the model. In Zhou and Zhong's study [3], based on passenger demands, the train plan problem is solved for a double-track high-speed line to minimize both the waiting time for high-speed trains and the total travel time for all trains, and a branch-and-bound algorithm with effective dominance rules is used to solve 
the bicriteria scheduling problem. Some works focus on periodic TTP, in which for every period (usually 1 hour) the timetable is repeated to assist passengers to remember and transfer between trains. A Periodic Event Scheduling Problem (PESP) model is applied by Liebchen and Möhring [4] and Kroon and Peeters [5]. We refer readers to the review by Cacchiani and Toth [6] of nominal and robust models and algorithms about TTP for further details. After the timetable is created, it should be evaluated to check its quality. In this field, much research focuses on the dynamic performance. In Huisamn and Boucherie's study [7], the delays caused by fast trains becoming caught behind slower ones are investigated, and a Markov chain is used to build a stochastic model capturing both scheduled and unscheduled train movements; the model allows the analysis of the running times for both fixed periodical timetables and long-term capacity calculation with random trains arriving. In Vromans et al's study [8], the reliability of the public railway system is examined by reducing the interdependency between trains and the homogeneity of the train timetable is evaluated. In Goverde's study [9], a Max-plus method is used to evaluate the stability of a periodic timetable by considering the delay propagation of the initial ones. These studies evaluate the train timetable from the point of view of effects among train paths, some of which call for such a specific condition as no overtaking is allowed (see, e.g., [7]) or a periodic timetable is used (see, e.g., [8]). In China, the evaluation emphasis differs. Usually, evaluation indexes are proposed to evaluate specific features like the passenger service level and infrastructure utilization level, and then subjective scores are given as inputs to describe how good the features are. In Peng et al.'s study [10], an index system including passenger service quality and dynamic performance as well as timetable production efficiency is built, and the timetable is evaluated with a Grey theory based on expert scores. In Zhu et al's study [11], the vague sets (see, e.g., Gau and Buehrer [12]) from the fuzzy sets are applied to another index system, which is proposed for the passengerdedicated line in terms of passenger transport demands, in order to deal with the experts' scores so that the timetable's evaluation can be gained.

The TTQ impacts the rail transport system directly, but it is difficult to compare the work skills of TMs. The major reason is that when a train path is set, one needs to consider several different constraints, usually including capacity constraints, which involve the running time of a train between stations, headway distances, crossover constraints, and so forth (see, e.g., [1]). Meanwhile, these constraints are caused by traffic flow, such as the number of trains and train stop patterns. Usually, we consider all the relevant factors such as capacity constraints and traffic flow as the background of the timetable. Once the timetable background is changed, the timetable features become incomparable. That is why when we try to evaluate a timetable's quality, a subjective input like an expert score is involved (see, e.g., $[10,11])$. However, the result of this method is unstable because it is based on the assumption that the experts are familiar with the timetable background. As mentioned before, when a TM adjusts a train path, since the constraints for the train paths will affect each other, the timetable background changes at the same time. Due to this consideration, there is no guarantee that the experts will give an objective score. For example, supposing we have 2 lines, one consisting of 100 trains with two speeds, $120 \mathrm{~km} / \mathrm{h}$ and $140 \mathrm{~km} / \mathrm{h}$, and the other consisting of 80 trains with four speeds, $160 \mathrm{~km} / \mathrm{h}, 140 \mathrm{~km} / \mathrm{h}, 120 \mathrm{~km} / \mathrm{h}$, and $80 \mathrm{~km} / \mathrm{h}$, given the different traffic flow and capacity constraints, it is hard to say which timetabling process is better. As the timetable background varies from line to line, the key point in evaluating the TTQ is to find a relative index which is still comparable even when the timetable background changes. Then, it will be possible to abandon the subjective inputs in the evaluation.

This paper introduces a TTQ evaluation method where the infrastructure features and traffic flows are no longer relevant. This allows us to determine the skill of the TMs even if the timetable background is different. We tested the method on different lines and obtained objective evaluation results without any subjective inputs, where the results showed the method to be effective and reasonable. As far as we know, this TTQ evaluation method is new.

The construction of this paper is as follows. In Section 2, we describe the problem and propose a train path efficiency index to evaluate the timetabling quality. In Section 3, we analyze the elements of the train path efficiency and give calculation methods. In Section 4, a solution model of train path efficiency based on Data Envelopment Analysis (DEA) is given, and the model is solved by linear programming. The method is verified with real-world timetables in China in Section 5, while in the final section some conclusions are given.

\section{Problem Description}

In TTP, the shortest running time between two stations is known as the ideal running time (see, e.g., [13]). A timetable with the ideal running time and minimum stop time is called an ideal timetable (see, e.g., [1]). In real-world cases, it is difficult to set an ideal timetable for every train, because some trains will conflict with others and violate the capacity constraints. To make a conflict-free timetable, some trains need to stop at a station longer or to slow down in the rail sector. In China, we allow TMs to modify the stop pattern and stop time of a train to meet the capacity constraints. Once the train type is given, the timetabling quality is affected mainly by the adjustment of the departure and arrival time instants as well as extra stop time.

An example is given to show how a train TM affects the timetabling quality in Figure 1: $\mathrm{T}$ and $\mathrm{K}$ are train types, where $\mathrm{T}$ is a long-distance train with a fixed departure time at station $\mathrm{A}$ and $\mathrm{K}$ is a short-distance train with a variable departure time at station $\mathrm{A}$, and the overtaking is due to capacity constraints. By modifying the departure time at station A, the improved train paths (expressed with dashed lines) improved the performances; for example, $\mathrm{K}^{\prime}$ has a better stop equilibrium because of the shorter average stop distance and $\mathrm{K} 2^{\prime}$ has less total travel time because some unnecessary stops are avoided.

From this simplified example, we can see that as we allow the TM to modify the departure and stop time at stations (at 


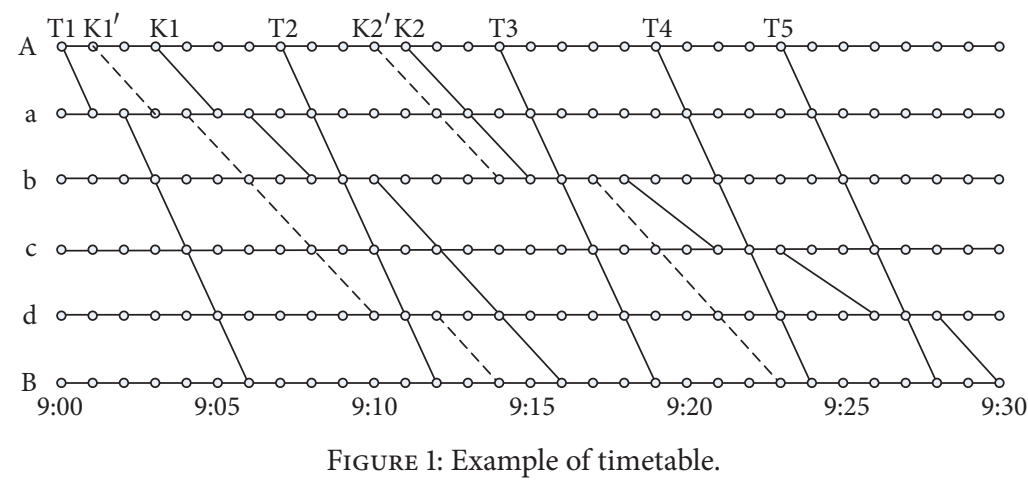

both the departure station and the intermediate stations), the train path performance can actually be improved. Thus, the TTQ, which indicates the TM's work skill, can be reflected through the adjustments of train paths. Because of the different speeds and stop patterns of trains, some stopping due to capacity constraints is inevitable, for example, if we imagine that we have a timetable that has scheduled lots of trains with different speeds, and it is obvious that the slow trains will be overtaken by fast trains and cause a slower speed feature. Yet, this overtaking is necessary; otherwise, no feasible solution could be found for the slow trains. Considering this, a good TM should try to modify the train path as follows: try to move the train path to see if this modification can avoid conflicts with other trains and meanwhile try to make the stop time more efficient, which means setting the stop time at a station to be as short as possible but respecting the minimum stop time constraints.

In Figure 1, we can see some examples of the modifications mentioned above. Once train $\mathrm{K} 1$ moved to $\mathrm{K}^{\prime}$, although the total stop time does not change, considering the stop distribution feature, the stops of $\mathrm{K}^{\prime}$ are more balanced. As for K1, which will make two stops, this will cause higher energy consumption because the train needs to accelerate and decelerate repeatedly. Also, once train $\mathrm{K} 2$ was moved to $\mathrm{K} 2$ ', an overtake is avoided and this improves the travel speed (due to the shorter total stop time). But remember, if we want to schedule train $\mathrm{K} 2$ without overtaking, no solution can be found (considering a certain departure time window, e.g., within 2 minutes before and after the departure time of K2). So, the stop caused by overtaking for train $\mathrm{K} 2$ is necessary and this should be considered when we evaluate the TM's work skill.

When we view a timetable that includes train paths which have different features such as stop patterns and speed, we can say that the TTQ is good if most stops of a train are necessary and balanced. Because the adjustments of train paths are done by TMs, it is natural that the train path efficiency can be used to evaluate the TTQ, which is also known as the TM work skill mentioned before.

If we consider a train path as a production unit, we can take a timetable as one production system which includes many production units with each unit having similar inputs and outputs. Thus, once we manage to find a way to obtain the efficiency of the units, we can analyze the TTQ objectively.
This proposal is reasonable because trains are scheduled one by one, attempting to find the best solution with respect to the formed structure (see, e.g., [1]). This will lead to a solution in which, for each train of the same type, the efficiency is as similar as possible. And a train path with lower efficiency will have worse quality.

In this paper, we propose a train path efficiency index that uses the concept of the production unit efficiency. Then, we use this train path efficiency index to evaluate the timetabling quality. To obtain the objective value of the train path efficiency, a Data Envelopment Analysis (DEA) method is applied. As the proposed index does not need subjective inputs, it is more reliable than other evaluation methods.

\section{Train Path Efficiency}

A train timetable contains several train paths, which define the location where a train is supposed to be at a given time instant. To schedule a train, two behaviors are involved: travel between stations and stops at stations. So, the rail line can be separated into two parts: the sector track between two stations and the station track at a station. Taking these as transport resources, each train path will then consume these transport resources. Then, a train has certain technical features like the stop time at a station, travel speed between two stations, and so forth. Taking these as technical productions, each train path will produce these technical productions at the same time. As a simple example, occupation of the sector track will produce travel speed. We define the ratio of production and resource as a kind of efficiency, in the case above, the ratio of travel speed and track occupation time of the sector, and then this efficiency is comparable without loss of generality. As mentioned, assuming that two trains are of the same type, if an extra buffer time is set for one of them, then the track consumption between two stations will increase and the travel speed production will decrease, which will cause a lower efficiency. This is because, for the same type, the extra buffer time is assumed to be the same for every train. For the occupation of the station track, consuming the resource will produce the stop time for checking of the rolling stock and passenger loading/alighting. If we suppose that the stop time is for passenger services, we can define another efficiency, which is the ratio of production and resource, specifically, of the effective stop time and station 
track occupation time. Remember that the TM can prolong the stop time of a train to resolve conflicts with other trains and that trains of the same type should have the same or nearly the same service level. If a train stops at a station longer than others, this train path will have a worse travel speed and lower efficiency.

Because of the capacity constraints and traffic flow, the stop pattern and extra buffer time are highly dependent on the timetable background. This makes it hard to find a certain solution that defines how good the performance a train path should be since the TTP problem is NP-hard itself (see Caprara et al. [1]). In this case, the evaluation of train path efficiency should be relative, which means that a given train path is relatively good compared with others. Because of this relative feature, the timetable background is involved in train path efficiency and makes the train path efficiency comparable. This relative feature is important when we try to evaluate the TTQ because, by applying the train path efficiency, we can abandon the subjective inputs in order to get a more reliable result.

Meanwhile, since the train path efficiency is relative, it is possible that the efficiency value is good, while for every train path, the absolute efficiency is low. But this case will not happen in practice because, as mentioned before, the timetabling process is to find the best solution for each train path with respect to the constraints. Therefore, the absolute low efficiency train path will never be selected and this avoids naturally the disadvantage of relative evaluation.

The display of train path not only affects the train path efficiency, but also has a strong impact on the dynamic features of the timetable such as its robustness and reliability. The dynamic aspect of the timetable is mainly about setting extra buffer time between train paths and using this extra buffer time to absorb possible delays in order to reduce the propagation. Usually, we set this extra buffer time according to the data that indicates where and when the delay will happen. We refer readers to Cacchiani and Toth [6] for further details over this problem. However, in this paper, we assume that the buffer time set is balanced, which means that the TMs set the buffer time equally between every train path. Thus, we can ignore the buffer time evaluation and the dynamic features of the timetable are therefore out of the scope of this paper.

To obtain the value of train path efficiency, one possible way is to give each element of resource and production a weight and then use the weighted ratio of productions and resources as the value of the train path efficiency.

Consider a technical output set $\Psi_{i}$ given out by train path $i$, known as production; $\Theta_{i}$ is a transport resource set consumed by train path $i$, known as resource. The efficiency of train path $i$ is defined as

$$
\eta_{i}=\frac{u \Psi_{i}}{v \Theta_{i}}, \quad 1 \leq i \leq N
$$

where $N$ is the number of train paths and $u$ and $v$ are the weight sets.

Although $\Psi_{i}$ and $\Theta_{i}$ depend on the traffic flow, infrastructure features, and so forth, the relative feature of the train path efficiency guarantees that its value is comparable even when the timetable background is different. By analyzing the train path efficiency in a timetable, we can evaluate the quality of the timetabling.

Notice that other train path efficiency criteria such as the ratio of loading/alighting volumes and stop time, the ratio of incomes, and costs of each train path could be proposed. However, in this paper, we focus on the technical features of the timetabling, so the service and economic features are not considered and will be surveyed in the future.

To get $\eta_{i}$, we need to analyze the transport resources and the technical productions of a train path. We propose two kinds of transport resource and four kinds of feedback production. Again, these proposals are from the technical point of view.

3.1. Train Path Transport Resource. A train path occupies the sector and station tracks, and we give the calculation method of them in this subsection.

3.1.1. Sector Track Occupation Time. Train path $i$ passes $n_{i}$ sectors, $d_{i s}$ is the length of sector $s\left(1 \leq s \leq n_{i}\right)$, and $v_{i s}$ is the speed of train path $i$ in sector $s$. The sector occupation time for train path $i$ is

$$
t_{\mathrm{sec}}^{i}=\sum_{s=1}^{n_{i}} \frac{d_{i s}}{v_{i s}} .
$$

3.1.2. Station Track Occupation Time. Train path $i$ stops at station $c_{i 1}, c_{i 2}, \ldots, c_{i j}, \ldots, c_{i m_{i} i}$; for each station, the station track occupation time starts from the signal that has been prepared and ends when the station throat is clear. So, it is longer than the actual stop time of the train at the station. The station track occupation time for train path $i$ at station $c_{i j}\left(1 \leq i \leq N, 1 \leq j \leq m \_i\right)$ is

$$
t_{\text {sta }}^{c_{i j}}=t_{\text {approach }}^{c_{i j}}+t_{\text {stop }}^{c_{i j}}+t_{\text {leave }}^{c_{i j}}
$$

where $t_{\text {approach }}^{c_{i j}}$ is the running time, from when the signal has been prepared for the train to enter the station $c_{i j}$ until the train arrives at station $c_{i j}$, which is

$$
t_{\mathrm{approach}}^{c_{i j}}=\frac{L_{\mathrm{break}}^{c_{i j}}+L_{\mathrm{pro}}^{c_{i j}}+L_{\mathrm{in}}^{c_{i j}}}{v_{\mathrm{st}}^{c_{i j}}}+t_{\mathrm{apr}}^{c_{i j}},
$$

where $L_{\text {break }}^{c_{i j}}$ is the breaking distance, $L_{\text {pro }}^{c_{i j}}$ is the protection distance, $L_{\text {in }}^{c_{i j}}$ is the distance from the signal to the stop point, $v_{\mathrm{st}}^{c_{i j}}$ is the average approaching speed until stopping, and $t_{\mathrm{apr}}^{c_{i j}}$ is the approaching time, which is the reaction time for the train driver to confirm the signal and respond with corresponding operations [14].

$t_{\text {stop }}^{c_{i j}}$ is the stop time for train path $i$ at station $c_{i j}$, given by

$$
t_{\text {stop }}^{c_{i j}}=t_{c_{i j}} b-t_{c_{i j}} a \text {, }
$$

where $t_{c_{i j}} a$ and $t_{c_{i j}} b$ are the arrival and departure time instants at station $c_{i j}$, respectively. 


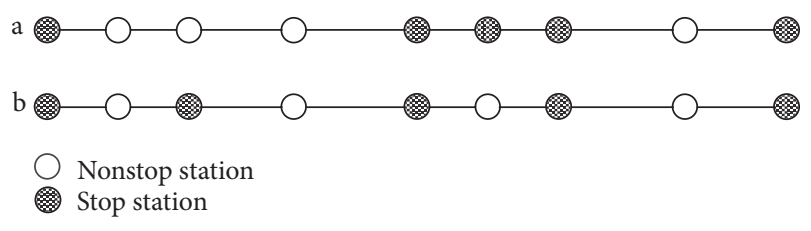

FIGURE 2: Stop distributions.

$t_{\text {leave }}^{c_{i j}}$ is the departure interval of train path $i$ at station $c_{i j}$; it starts when the departure signal is ready and ends when the train has left the station and the station track can be used by other train paths, which is

$$
t_{\text {leave }}^{c_{i j}}=\frac{L_{\text {signal }}^{c_{i j}}+L_{\text {block }}^{c_{i j}}+L_{\text {train }}^{i}}{v_{\text {le }}^{c_{i j}}}+t_{\text {lev }}^{c_{i j}},
$$

where $L_{\text {signal }}^{c_{i j}}$ is the distance from the stop point to the departure signal, $L_{\text {block }}^{c_{i j}}$ is the leaving block length, $L_{\text {train }}^{i}$ is the train length, $v_{\text {le }}^{c_{i j}}$ is the average leaving speed, and $t_{\text {lev }}^{c_{i j}}$ is the departing time, which is the reaction time for the train driver to confirm the signal and respond with corresponding operations [14].

Because the signal set and station tracks are not always the same for every station, the track occupation time may differ from station to station. The total station track occupation time of train path $i$ is

$$
\begin{aligned}
t_{\mathrm{sta}}^{i} & =\sum_{j=1}^{m_{-} i} t_{\mathrm{sta}}^{c_{j j}}=\sum_{j=1}^{m_{-} i}\left(\frac{L_{\text {break }}^{c_{i j}}+L_{\text {pro }}^{c_{i j}}+L_{\text {in }}^{c_{i j}}}{v_{\mathrm{st}}^{c_{i j}}}+t_{\mathrm{apr}}^{c_{i j}}+t_{c_{i j}} b\right. \\
& \left.-t_{c_{i j}} a+\frac{L_{\text {signal }}^{c_{j j}}+L_{\text {block }}^{c_{i j}}+L_{\text {train }}^{i}}{v_{\text {le }}^{c_{i j}}}+t_{\text {lev }}^{c_{i j}}\right) .
\end{aligned}
$$

3.2. Train Path Technical Production. The train path technical production includes the efficient stop time and several kinds of speed. Once a train stops at a station, there is an ideal stop time which is the minimum stop time with respect to the capacity constraints or passenger loading/alighting stop time limitations. But usually it is hard to set every train path to have the ideal stop time at each stop. Generally, we believe that the ideal stop time is efficient, and the stop time beyond ideal stop time is inefficient. So, we propose the efficient stop time in order to check the adjustments of train paths. Considering that each train path should have an equilibrium stop distribution and that the TMs can adjust the stop pattern, we propose different kinds of speed to evaluate the stop pattern. An example of two stop patterns with the same number of stops is given in Figure 2. In the example, plan (a) is worse because the stops are bunched and this will increase the energy consumption for frequent acceleration and deceleration.

We propose four kinds of speed as the technical productions of a train path, which are the efficient stop time, average running speed, average travel speed, and travel speed.
3.2.1. Efficient Stop Time. For train path $i$ at station $c_{i j}$, the minimum stop time is determined by the passenger loading/alighting needs or the capacity constraints. As the minimum stop time differs from stations and trains, we use $t s\left(c_{i j}\right)$ to express the overall efficient stop time of train path $i$ at station $c_{i j}$, so the efficiency stop time for train path $i$ is

$$
t_{\mathrm{ef}}^{i}=\sum_{j=1}^{m-i} t s\left(c_{i j}\right)
$$

3.2.2. Average Running Speed. For train path $i$ in sector $s(1 \leq$ $s \leq n_{-} i$ ) with the length $d_{i s}$, there are a maximum track speed $v_{\text {lim }}^{s}$, maximum locomotive speed $v_{\text {limloc }}$, and maximum vehicle speed $v_{\text {limvie }}$. Because the minimum running time in sector $s$ is limited by the maximum possible speed for train path $i$ in sector $s$, the average running speed is

$$
v_{\text {sec }}^{i}=\frac{1}{n_{i}} \sum_{s=1}^{n_{i}} \frac{d_{i s}}{\max \left(d_{i s} / v_{\text {lim }}^{s}, d_{i s} / v_{\text {limloc }}, d_{i s} / v_{\text {limvie }}\right)} .
$$

3.2.3. Average Travel Speed. For train path $i$ in sector $s(1 \leq$ $s \leq n_{-} i$ ) with the length $d_{i s}, v_{i s}$ is the running speed of train path $i$ in sector $s$, and there are an acceleration/deceleration extra time $t_{i s}^{q t}$ and an extra running time $t_{i s}^{e}$.

Whether or not an acceleration/deceleration extra time is added depends on whether or not the train path $i$ stops at the start/end station of the sectors. If the train path $i$ stops at the start station of sector $s$, an acceleration extra time $t_{\text {stand }}^{q}$ should be added; if the train path $i$ stops at the end station of sector $s$, a deceleration extra time $t_{\text {stand }}^{t}$ should be added. Thus, $t_{i s}^{q t}$ is

$$
t_{\text {is }}^{q t}=\lambda_{1} t_{\text {stand }}^{q}+\lambda_{2} t_{\text {stand }}^{t} .
$$

In (10), $\lambda_{1}$ and $\lambda_{2}$ are binary variables, equal to 1 if the train stops at the corresponding stations mentioned above. The extra running time $t_{i s}^{e}$ is due to the buffer times. The average travel speed is

$$
\begin{aligned}
v_{\mathrm{ave}}^{i} & =\frac{1}{n_{i}} \sum_{s=1}^{n_{i}} \frac{d_{i s}}{d_{i s} / v_{i s}+t_{i s}^{e}+t_{i s}^{q t}} \\
& =\frac{1}{n_{i}} \sum_{s=1}^{n_{i}} \frac{d_{i s} v_{i s}}{d_{i s}+v_{i s}\left(t_{i s}^{e}+t_{i s}^{q t}\right)} .
\end{aligned}
$$

3.2.4. Travel Speed. For train path $i$, this is determined by the total travel distance and total travel time, which is

$$
v_{\text {trv }}^{i}=\frac{\sum_{s=1}^{n_{i}} d_{i s}}{t_{c_{i i_{i} i} a-t_{c_{i 1}} b} .}
$$

Here, we give some explanations of the proposed speeds. Formula (9) checks if the rolling stock plan is appropriate. The rolling stock should be used according to the type of train. This means that a high-speed rolling stock unit should be assigned to a high-speed train and vice versa. Using a fast train unit to run a slow train is a waste and should be avoided. 
If faster rolling stock is assigned to a slow train, compared with other train paths with the same average running speed, the track occupation time will be larger. This will cause the transport resource consumption to increase and reduce the train path efficiency. Formula (11) checks if the stop is at equilibrium. Given a certain number of stops, the average travel speed will be lower if the stops are concentrated. Moreover, extra time should be added equally to every train path as well, or else some train paths will have lower performance if more extra time is assigned. For two trains of the same type, if the number of stops is the same, the train path with an equilibrium stop pattern will have a higher average travel speed; if the number of stops and the stop patterns are the same, the train path with less extra time will have a higher travel speed.

\section{Train Path Efficiency Calculation and Timetabling Quality Evaluation}

The value of train path efficiency is determined not only by the train path itself but also by the other train paths in a timetable. This is because one train path becomes a constraint for the others once it has been scheduled. Good TTQ means a highly balanced train path efficiency value for all train paths. As the proposed train path efficiency is a weighted ratio, the value of train path efficiency is decided by the weight parameters. We need to set an objective weight for every element of the train path efficiency in order to get an objective result. In this sector, we introduce a Data Envelopment Analysis (DEA) method to get an objective value of the train path efficiency. The benefit of this application is that DEA allows us to calculate the relative efficiency of a production unit in a production system, while all the parameters are decided by the feature of the production system itself. This means that no subjective input is required and can lead to a more reliable result.

4.1. Index Definition and Solution Method Based on DEA. Taking the train path as a production unit, each train path $i$ uses $\varphi_{i}^{p}$ resources with positive numbers $\alpha_{i}^{p}$ (value of item $p$ used by $i$ ) to produce $\theta_{i}^{k}$ productions valued by $\beta_{i}^{k}$ (value of item $k$ produced by $i$ ); suppose we have $w$ kinds of output and $q$ kinds of input, and the efficiency of train path $i$ is defined as

$$
\eta_{i}=\frac{\sum_{p=1}^{w} \alpha_{i}^{p} \varphi_{i}^{p}}{\sum_{k=1}^{q} \beta_{i}^{k} \theta_{i}^{k}}, \quad \alpha_{i}^{p}, \beta_{i}^{k}>0 .
$$

It can be solved using DEA method in a short computation time.

DEA was proposed by Charnes, Cooper, and Rhodes. It is an efficient way to qualify the relative efficiency by searching for the best virtual producer using linear programming models [15]. For a group of producers that consume the same kinds of resources and produce the same kinds of productions,
DEA allows us to evaluate the efficiency of each producer with the model below:

$$
\begin{aligned}
e_{i}= & \frac{\sum_{p=1}^{w} \alpha_{i}^{p} \varphi_{i}^{p}}{\sum_{k=1}^{q} \beta_{i}^{k} \theta_{i}^{k}} \\
\text { s.t. } & \frac{\alpha_{i}^{p} \varphi_{i}^{p}}{\beta_{i}^{k} \theta_{i}^{k}} \leq 1, \\
& \quad i=1, \ldots, n ; p=1, \ldots, w ; k=1, \ldots, q
\end{aligned}
$$

$\alpha \geq 0$,

$\beta \geq 0$.

For producer $i$, the model finds a virtual producer with respect to the global productivity by solving $n$ linear programming models and then uses parameters $\alpha_{i}^{p}$ and $\beta_{i}^{k}$ of the virtual producer to get the relative efficiency of producer $i$. We can see that as no subjective input is needed for the process, the result is therefore objective. We refer readers to Charnes et al. [15] for a better knowledge of the model above.

4.2. Solution Model of Train Path Efficiency. As a multi-inputoutput system efficiency evaluation problem, we apply DEA to build the train path efficiency solution model:

$$
\begin{aligned}
\eta_{i_{0}}=\max & \frac{u_{1} t_{\mathrm{ef}}^{i_{0}}+u_{2} v_{\mathrm{sec}}^{i_{0}}+u_{3} v_{\mathrm{ave}}^{i_{0}}+u_{4} v_{\mathrm{trv}}^{i_{0}}}{\mu_{1} t_{\mathrm{sec}}^{i_{0}}+\mu_{2} t_{\mathrm{sta}}^{i_{0}}} \\
\text { s.t. } & \frac{u_{1} t_{\mathrm{ef}}^{i}+u_{2} v_{\mathrm{sec}}^{i}+u_{3} v_{\mathrm{ave}}^{i}+u_{4} v_{\mathrm{trv}}^{i}}{\mu_{1} t_{\mathrm{sec}}^{i}+\mu_{2} t_{\mathrm{sta}}^{i}} \leq 1, \\
& \\
u \geq 0, & i=1, \ldots, N \\
& \mu \geq 0,
\end{aligned}
$$

where $\eta_{i_{0}}\left(1 \leq i_{0} \leq N\right)$ is the efficiency value of train path $i_{0}$ proposed in (1) and $u_{1}, u_{2}, u_{3}, u_{4}$ and $\mu_{1}, \mu_{2}$ are parameters decided by the linear programming model.

Set $U=\left(u_{1}, u_{2}, u_{3}, u_{4}\right)^{T}, M=\left(\mu_{1}, \mu_{2}\right)^{T}, X_{i}=\left(t_{\text {sec }}^{i}, t_{\text {sta }}^{i}\right)^{T}$, $Y_{i}=\left(v_{\text {sec }}^{i}, v_{\text {ave }}^{i}, v_{\text {trv }}^{i}\right)^{T}, \alpha=1 / M^{T} X_{i_{0}}, \beta=\alpha M, \gamma=\alpha u$, using the Charnes-Cooper transformation to simplify the model $[15,16]$ :

$$
\begin{aligned}
\eta_{i_{0}}=\max & \gamma^{T} Y_{i_{0}} \\
\text { s.t. } & \beta^{T} X_{i}-\gamma^{T} Y_{i} \geq 0, \quad i=1,2, \ldots, N \\
& \beta^{T} X_{i_{0}}=1 \\
& \forall \beta \geq 0, \\
& \forall \gamma \geq 0 .
\end{aligned}
$$

By solving $N$ linear programming models, we can get the train path efficiency value set $H=\left(\eta_{1}, \eta_{2}, \ldots, \eta_{N}\right)$ and evaluate the timetabling quality. 
4.3. Evaluation of Train Timetabling Quality. We evaluate the TTQ based on the train path efficiency value set $H=$ $\left(\eta_{1}, \eta_{2}, \ldots, \eta_{N}\right)$. As mentioned before, the average train path efficiency can be used to evaluate the TTQ; therefore, we use the train path efficiency expectation (TEE)

$$
E_{\eta}=\frac{1}{N} \sum_{i=1}^{N} \eta_{i}
$$

as the evaluation results of TTQ.

In addition, let

$$
\varphi\left(\eta_{i}\right)= \begin{cases}1, & \eta_{i} \in\left(\eta_{s}, \eta_{s}-\tau\right) \\ 0, & \text { else }\end{cases}
$$

where $\eta_{s}$ is the given train path efficiency value; we introduce a train path efficient distribution function (EDF) with interval $\tau$ to describe the distribution of train path efficiency of the timetable:

$$
f\left(\eta_{s}, \eta_{s}-\tau\right)=\frac{\sum_{i=1}^{N} \varphi\left(\eta_{i}\right)}{N} \times 100 \% .
$$

The TEE shows the average level of train path efficiency, which is in positive correlation with TTQ. It is obvious that a timetable with a higher TEE has a better TTQ. Meanwhile, EDF gives the efficiency distribution, which can help us to analyze the train path efficiency distribution of a timetable in detail.

\section{Case Study}

In this section, we use real-world timetables in China to verify our TTQ evaluation method. As mentioned before, the relative feature of train path efficiency is the key to evaluate the TTQ. In this section, we first show the relative feature of the train path efficiency. Then, we evaluate the TTQ of timetables with different backgrounds and compare their TTQ to show that our method can give objective results.

5.1. Train Path Efficiency Feature Test. The first case is the $119 \mathrm{~km}$ Shibantan-to-Xinqiao line with 4 intermediate stations. The maximum track speed is $200 \mathrm{~km} / \mathrm{h}$ and 112 trains of four types are scheduled. We use $t_{\text {stand }}^{q}$ and $t_{\text {stand }}^{t}$ to represent $t_{\text {approach }}^{c_{i j}}$ and $t_{\text {leave }}^{c_{i j}}$ as an approximation. Other parameters are as follows: $t_{\text {stand }}^{q}=t_{\text {stand }}^{t}=2 \mathrm{~min}$. Because $t_{\text {ef }}^{i}$ is highly dependent on the number of passengers that are loading/alighting as well as the constraints caused by overtaking, in the test, we simplify $t_{\text {ef }}^{i}$ cases as below:

If the stop is for passenger loading/alighting, then set $t_{\text {ef }}^{i}$ as the actual stop time.

If the stop is due to a train overtaking, then $t_{\mathrm{ef}}^{i}$ is set to $9 \mathrm{~min}$, and if there is more than one train overtaking, $t_{\mathrm{ef}}^{i}$ is set to $(9+5(k-1)) \mathrm{min}$, where $k$ is the number of trains that are overtaking.

If the stop involves both of the above, then set $t_{\mathrm{ef}}^{i}$ has the largest value.
The model is solved by Lingo 11 within 1 second on a Win7 64-bit 8 GB Core 2 personal computer. We report the train path efficiency value with the total number of stops (see Table 1) and the layout of travel speed with efficiency value to express the relative feature (see Figure 3), where the down trains run from Xinqiao to Shibantan and the up trains run in the opposite direction. The TEE is 0.8412 , and a DEF with interval 0.1 is given (see Table 2).

In this case, the value of train path efficiency is not relevant to the train type, which means that although the train speeds are different, their train path efficiency is comparable. In fact, the computing results also show that the train path efficiency is not relative to the infrastructure features either (shown in the next test case). As expected, since the train path efficiency is objective, we can evaluate the TTQ more convincingly.

For fast trains (type D), the stop affects the train path efficiency significantly. One more stop will cause the train path efficiency to drop greatly, for example, D5107 and D5109 (bold trains in Table 1). Given that the nonstop travel time for a fast train is $38 \mathrm{~min} 25 \mathrm{sec}$, one stop will increase the travel time by $15.6 \%$ ( $6 \mathrm{~min}$ ). This means that fast trains are sensitive to the total travel time. For slow trains (types T, K, and X), the average stop time is $17 \mathrm{~min} 10 \mathrm{sec}$, while the average travel time is $1 \mathrm{~h} 27 \mathrm{~min} 15 \mathrm{sec}$. Due to the capacity constraints, they have to stop to let fast trains overtake. The increase of travel time caused by the stop is $19.54 \%$. This means that slow trains are less sensitive to the total travel time than fast trains (see Figure 3).

Considering the sensitivity differences, it is better to schedule the fast train with fewer stops. This conclusion is logical because as the travel time between stations decreases, the train is more competitive with different means of transport. We would like to mention here that because the train path efficiency is a relative value, if most fast trains stop a lot, the relative value will not be impacted. This shows how the train path efficiency reflects the train plan and makes the result more reliable.

In the case investigated, over $98 \%$ of trains have an efficiency value greater than 0.8 . But some slow trains have low efficiency because of their long stop times (see Table 3). From this result, we can conclude that, compared with the efficient stop time, a longer stop will cause a decrease in train path efficiency.

The train path efficiency distinguishes the stop distribution differences as expected. Taking trains K587 and K697 as an example, the total stop times are $32 \mathrm{~min}$ and $34 \mathrm{~min}$. Train K587 stops twice while train K697 stops only once, and the lower average travel speed leads to a worse efficiency value for K587. In another result, T248 has a higher train path efficiency than K1094 although the total stop time is longer. This is because T248 has a better stop plan (see Figure 4). This means that the stop distribution is more important than the total stop time for this case. But note that this conclusion is based on a specific case and that it is possible that the total stop time is more important than the stop distribution in other cases. 
TABLE 1: Train path efficiency value.

(a) Trains from Xinqiao to Shibantan

\begin{tabular}{lcccccccccccccc}
\hline Train number & $\mathrm{D} 5102$ & $\mathrm{D} 5104$ & $\mathrm{D} 5106$ & $\mathrm{D} 5108$ & $\mathrm{D} 5110$ & $\mathrm{D} 5112$ & $\mathrm{D} 5114$ & $\mathrm{D} 5116$ & $\mathrm{D} 5118$ & $\mathrm{D} 5120$ & $\mathrm{D} 5122$ & $\mathrm{D} 5124$ & $\mathrm{D} 5126$ & $\mathrm{D} 5128$ \\
$\eta_{i}$ & 0.9935 & 0.9935 & 0.8442 & 0.9935 & 0.8503 & 0.9935 & 0.8503 & 0.9935 & 0.9935 & 0.9935 & 0.9723 & 0.9935 & 0.9935 & 0.9935 \\
Number of stops & 0 & 0 & 1 & 0 & 1 & 0 & 1 & 0 & 0 & 0 & 0 & 0 & 0 & 0 \\
Train number & $\mathrm{D} 5130$ & $\mathrm{D} 5132$ & $\mathrm{D} 5134$ & $\mathrm{D} 5136$ & $\mathrm{D} 5162$ & $\mathrm{D} 5164$ & $\mathrm{D} 5166$ & $\mathrm{D} 5168$ & $\mathrm{D} 5170$ & $\mathrm{D} 5174$ & $\mathrm{D} 5182$ & $\mathrm{D} 5184$ & $\mathrm{D} 5186$ & $\mathrm{D} 5188$ \\
$\eta_{i}$ & 0.9935 & 0.9935 & 0.9828 & 0.9935 & 0.8442 & 0.8442 & 0.9935 & 0.9935 & 0.9935 & 0.9935 & 0.9935 & 0.9935 & 0.9535 & 0.9935 \\
Number of stops & 0 & 0 & 0 & 0 & 1 & 1 & 0 & 0 & 0 & 0 & 0 & 0 & 0 & 0 \\
Train number & $\mathrm{D} 5190$ & $\mathrm{D} 5196$ & $\mathrm{D} 5198$ & $\mathrm{D} 55522$ & $\mathrm{Z} 124$ & $\mathrm{~T} 126$ & $\mathrm{~T} 248$ & $\mathrm{~T} 252$ & $\mathrm{~T} 8900$ & $\mathrm{~K} 118$ & $\mathrm{~K} 352$ & $\mathrm{~K} 424$ & $\mathrm{~K} 488$ & $\mathrm{~K} 530$ \\
$\eta_{i}$ & 0.9892 & 0.9892 & 0.9935 & 0.9935 & 0.9304 & 0.9545 & 0.8820 & 0.8974 & 0.8974 & 0.9545 & 0.9545 & 0.8820 & 0.9545 & 0.9545 \\
Number of stops & 0 & 0 & 0 & 0 & 0 & 0 & 1 & 1 & 1 & 0 & 0 & 1 & 0 & 0 \\
Train number & $\mathrm{K} 578$ & $\mathrm{~K} 588$ & $\mathrm{~K} 652$ & $\mathrm{~K} 678$ & $\mathrm{~K} 698$ & $\mathrm{~K} 724$ & $\mathrm{~K} 746$ & $\mathrm{~K} 788$ & $\mathrm{~K} 818$ & $\mathrm{~K} 1094$ & $\mathrm{~K} 1158$ & $\mathrm{~K} 1196$ & $\mathrm{~K} 1258$ & $\mathrm{X} 228$ \\
Number of stops & 1 & 0 & 1 & 1 & 1 & 1 & 1 & 1 & 1 & 0 & 0 & 0 & 0 & 0 \\
$\eta_{i}$ & 0.9111 & 0.9266 & 0.9219 & 0.8694 & 0.8694 & 0.8968 & 0.9025 & 0.8694 & 0.9545 & 0.8488 & 0.9864 & 0.9545 & 0.9545 & 0.7319 \\
\hline
\end{tabular}

(b) Trains from Shibantan to Xinqiao

\begin{tabular}{lccccccccccccccc}
\hline Train number & $\mathrm{D} 5101$ & $\mathrm{D} 5103$ & $\mathrm{D} 5105$ & $\mathrm{D} 5107$ & $\mathrm{D} 5109$ & $\mathrm{D} 5111$ & $\mathrm{D} 5113$ & $\mathrm{D} 5115$ & $\mathrm{D} 5117$ & $\mathrm{D} 5119$ & D5121 & D5123 & D5125 & D5127 \\
$\eta_{i}$ & 0.9913 & 0.9913 & 0.8413 & 0.9913 & 0.8520 & 0.9913 & 0.8535 & 0.9913 & 0.9913 & 0.9913 & 0.9913 & 0.8492 & 0.8413 & 0.9913 \\
Number of stops & 0 & 0 & 1 & 0 & 1 & 0 & 1 & 0 & 0 & 0 & 0 & 1 & 1 & 0 \\
Train number & $\mathrm{D} 5129$ & $\mathrm{D} 5131$ & $\mathrm{D} 5133$ & $\mathrm{D} 5135$ & $\mathrm{D} 5161$ & $\mathrm{D} 5163$ & $\mathrm{D} 5165$ & $\mathrm{D} 5167$ & $\mathrm{D} 5169$ & $\mathrm{D} 5173$ & $\mathrm{D} 5181$ & D5183 & D5185 & D5187 \\
$\eta_{i}$ & 0.9913 & 0.9913 & 0.9913 & 0.9913 & 0.8520 & 1.0000 & 0.9913 & 0.8423 & 0.9913 & 0.9913 & 0.9913 & 0.9913 & 0.9517 & 0.9913 \\
Number of stops & 0 & 0 & 0 & 0 & 1 & 0 & 0 & 1 & 0 & 0 & 0 & 0 & 0 & 0 \\
Train number & $\mathrm{D} 5189$ & $\mathrm{D} 5195$ & $\mathrm{D} 5197$ & $\mathrm{D} 55519$ & $\mathrm{Z} 123$ & $\mathrm{~T} 125$ & $\mathrm{~T} 247$ & $\mathrm{~T} 251$ & $\mathrm{~T} 8899$ & $\mathrm{~K} 117$ & $\mathrm{~K} 351$ & $\mathrm{~K} 423$ & $\mathrm{~K} 487$ & $\mathrm{~K} 529$ \\
$\eta_{i}$ & 0.9913 & 0.9709 & 0.8444 & 0.9662 & 0.9521 & 0.9109 & 0.9617 & 0.9473 & 0.9617 & 0.9765 & 0.8190 & 0.8968 & 0.9473 & 0.9617 \\
Number of stops & 0 & 0 & 1 & 0 & 0 & 1 & 0 & 0 & 0 & 0 & 0 & 1 & 0 & 0 \\
Train number & $\mathrm{K} 577$ & $\mathrm{~K} 587$ & $\mathrm{~K} 651$ & $\mathrm{~K} 677$ & $\mathrm{~K} 697$ & $\mathrm{~K} 723$ & $\mathrm{~K} 745$ & $\mathrm{~K} 787$ & $\mathrm{~K} 817$ & $\mathrm{~K} 1093$ & $\mathrm{~K} 1157$ & $\mathrm{~K} 1195$ & $\mathrm{~K} 1257$ & $\mathrm{X} 227$ \\
$\eta_{i}$ & 0.8111 & 0.8818 & 0.9182 & 0.9260 & 0.9045 & 0.9617 & 0.9617 & 0.8968 & 0.9155 & 0.9473 & 0.8553 & 0.9617 & 0.9045 & 0.6157 \\
Number of stops & 4 & 2 & 1 & 2 & 1 & 0 & 0 & 1 & 1 & 0 & 2 & 0 & 1 & 2 \\
\hline
\end{tabular}

5.2. Timetabling Quality Comparison Test. The second case is the $393 \mathrm{~km}$, double-track, mixed traffic Qingdao-to-Jinan line with a maximum track speed of $200 \mathrm{~km} / \mathrm{h}$. We use the 2012 and 2013 timetables with different numbers of trains, and the parameters are the same as the first case. Note that as the number and composition of trains are different for these two timetables, based on the existing evaluation method, we cannot say which one has a better TTQ, while subjective input like an expert score cannot guarantee a reliable result either. We use the train path efficiency proposed to evaluate the TTQ of these two timetables. All results are computed within 4 seconds on the same computer. As before, we report the TEE as well as the EDF with a 0.1 interval as Table 4 shows.

As mentioned in the first case, because the timetable background has changed, the train path efficiency value shows its relative feature since the values of train path efficiency are in the same region. Again, this proves that we can use this train path efficiency to evaluate the TTQ without considering the timetable background.

Compared with the 2012 timetable, in the 2013 timetable, 15 more trains are scheduled, and the TEE decreased by 0.0065 . We can conclude that the timetabling quality is worse for 2013. One possible reason is that as the number of trains increased, the line became more congested. Because of the short-sighted nature of manual timetabling, it is difficult to find an optimized path for every train. Therefore, automatic timetabling should be applied for the case of congestion.

If we compare the evaluation results of the three cases, we will find that the timetables of the Qingdao-to-Jinan line have a higher TEE. This means that the TTQ of Qingdaoto-Jinan line is higher even though this line has a more congested traffic flow. This indicates that the TM in charge of the Qingdao-to-Jinan line is more skilled at his work.

The main reason why the Shibantan-to-Xinqiao line has a worse TTQ is that many trains have to make quite long stops, as shown in Table 3. This makes the stop time efficiency worse and means that the timetable could be optimized by adjusting some of the train paths, although the TM has not done this. So, the skill level of the TM in charge of the line of the first case is worse.

\section{Conclusions}

There is little research on the evaluation of timetabling quality. In this paper, we propose a relative train path efficiency index, and the value of train path efficiency is computed by applying DEA method. The timetabling quality was evaluated by the statistical analysis of the train path efficiency value. The method was verified by real-world train timetables in China and the results showed that it is practical. 


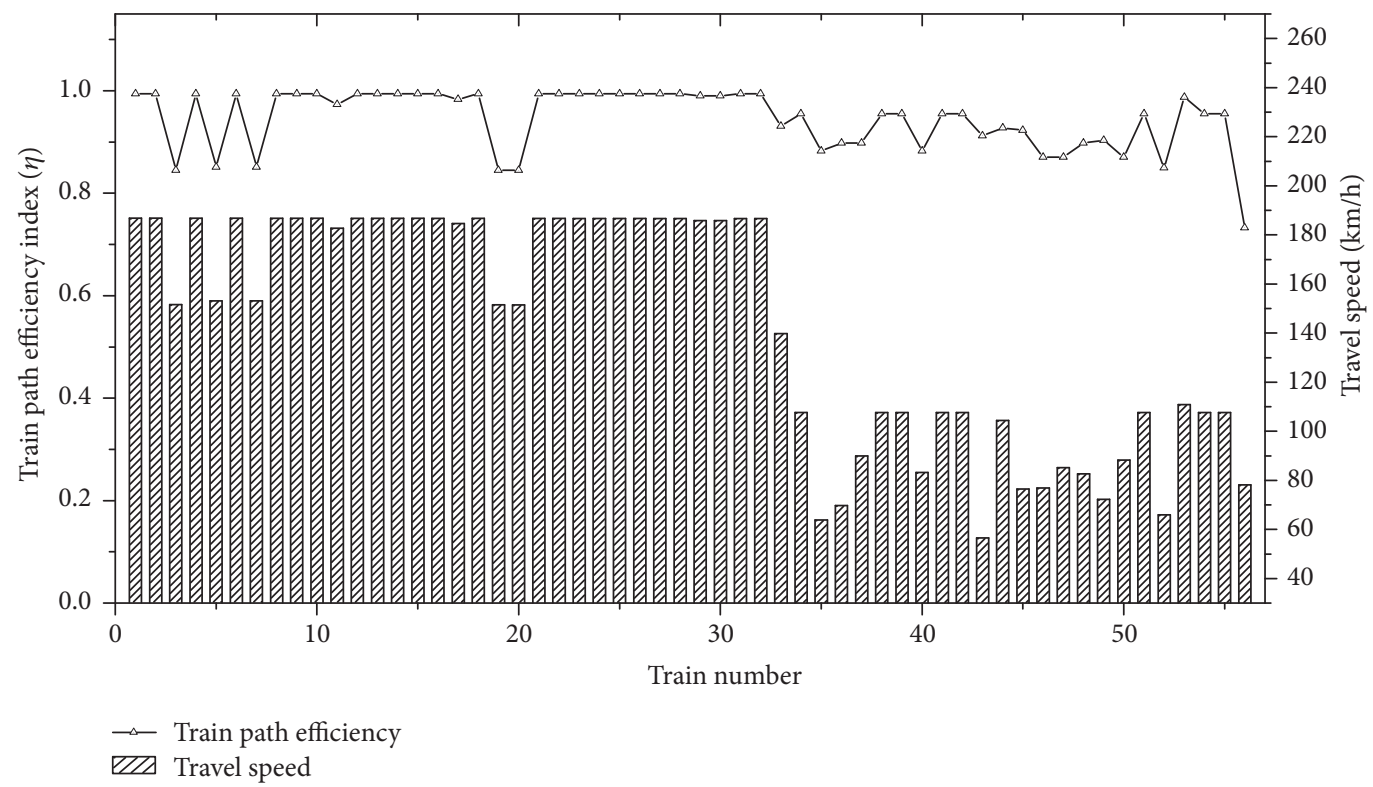

(a) Up train travel speed-efficiency index layout

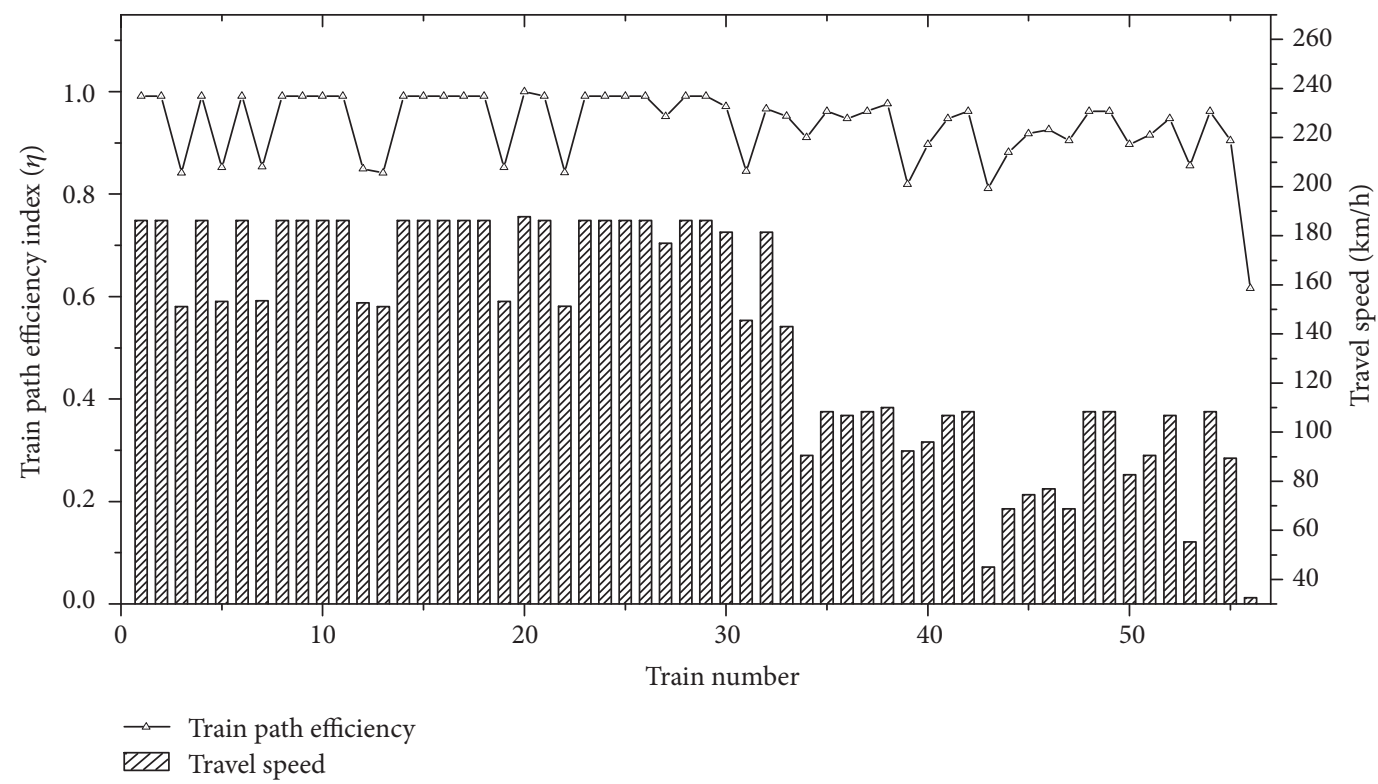

(b) Down train travel speed-efficiency index layout

FIgURE 3: Train path travel speed-efficiency value layout.

TABLE 2: Efficient distribution function (EDF) with 0.1 interval.

\begin{tabular}{lccccc}
\hline EDF & $(1,0.9)$ & $(0.9,0.8)$ & $(0.8,0.7)$ & $(0.7,0.6)$ & $(0.6,0)$ \\
Number of trains & 82 & 28 & 1 & 1 & 0 \\
Percentage & $73.21 \%$ & $25 \%$ & $0.89 \%$ & $0.89 \%$ & - \\
\hline
\end{tabular}

TABLE 3: Trains with stop time over $30 \mathrm{~min}$.

\begin{tabular}{lcccccccccc}
\hline Train number & K577 & K587 & K697 & K1157 & X227 & T248 & T252 & K578 & K788 & K1094 \\
Stop time & 1h $18 \mathrm{~min}$ & $32 \mathrm{~min}$ & $34 \mathrm{~min}$ & $56 \mathrm{~min}$ & $1 \mathrm{~h} 53 \mathrm{~min}$ & $40 \mathrm{~min}$ & $33 \mathrm{~min}$ & $57 \mathrm{~min}$ & $30 \mathrm{~min}$ & $35 \mathrm{~min}$ \\
Train path efficiency & 0.8111 & 0.8818 & 0.9045 & 0.8553 & 0.6157 & 0.8820 & 0.8974 & 0.9111 & 0.8694 & 0.8488 \\
Number of stops & 4 & 2 & 1 & 2 & 2 & 2 & 1 & 1 & 2 \\
\hline
\end{tabular}




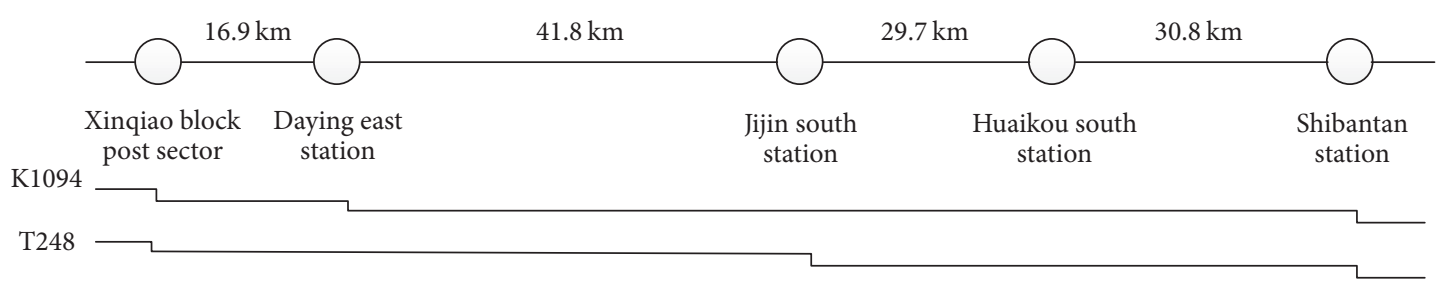

FIGURE 4: Station distributions.

TABLE 4: Evaluation results of Qingdao-to-Jinan line.

\begin{tabular}{|c|c|c|c|c|c|c|c|c|}
\hline \multicolumn{9}{|c|}{2013 timetable (186 trains: 76 high-speed trains, 4 express trains, and 106 normal trains) } \\
\hline TEE & TEV & EDF & $(1,0.9)$ & $(0.9,0.8)$ & $(0.8,0.7)$ & $(0.7,0.6)$ & $(0.6,0.5)$ & $(0.5,0.4)$ \\
\hline \multirow{2}{*}{0.9140} & \multirow{2}{*}{0.0545} & Number of trains & 7 & 92 & 60 & 14 & 10 & 3 \\
\hline & & Percentage & $3.76 \%$ & $49.46 \%$ & $32.26 \%$ & $7.53 \%$ & $5.38 \%$ & $1.61 \%$ \\
\hline \multicolumn{9}{|c|}{2012 timetable (171 trains: 60 high-speed trains, 2 express trains, and 109 normal trains) } \\
\hline TEE & TEV & EDF & $(1,0.9)$ & $(0.9,0.8)$ & $(0.8,0.7)$ & $(0.7,0.6)$ & $(0.6,0.5)$ & $(0.5,0.4)$ \\
\hline \multirow{2}{*}{0.9205} & \multirow{2}{*}{0.0586} & Number of trains & 8 & 87 & 52 & 12 & 10 & 2 \\
\hline & & Percentage & $4.68 \%$ & $50.88 \%$ & $30.41 \%$ & $7.02 \%$ & $5.85 \%$ & $1.17 \%$ \\
\hline
\end{tabular}

Further research will specify the efficiency part of the total stop time and verify the train path efficiency at the passenger service level. Another possibility is the application of sensitivity analysis to identify possible improvements of the train path efficiency.

\section{Conflicts of Interest}

The authors declare that there are no conflicts of interest regarding the publication of this paper.

\section{Acknowledgments}

The first author would like to express gratitude for the support from the National Railway Train Diagram Researching and Training Center, Southwest Jiaotong University, and the support of the Chinese Natural Science Foundation under Grant no. 61403317 and Technology Department of China Railway Cooperation under Grants nos. 2013X010-A and 2014X004D. The authors also appreciate the valuable comments from Dr. V. Cacchiani, DEI, University of Bologna.

\section{References}

[1] A. Caprara, M. Fischetti, and P. Toth, "Modeling and solving the train timetabling problem," Operations Research, vol. 50, no. 5, pp. 851-861, 2002.

[2] U. Brännlund, P. O. Lindberg, A. S. Nou, and J.-E. Nilsson, "Railway timetabling using lagrangian relaxation," Transportation Science, vol. 32, no. 4, pp. 358-369, 1998.

[3] X. Zhou and M. Zhong, "Bicriteria train scheduling for highspeed passenger railroad planning applications," European Journal of Operational Research, vol. 167, no. 3, pp. 752-771, 2005.

[4] C. Liebchen and R. H. Möhring, "A case study in periodic timetabling," Electronic Notes in Theoretical Computer Science, vol. 66 , no. 6 , pp. 18-31, 2002.
[5] L. G. Kroon and L. W. P. Peeters, "A variable trip time model for cyclic railway timetabling," Transportation Science, vol. 37, no. 2, pp. 198-212, 2003.

[6] V. Cacchiani and P. Toth, "Nominal and robust train timetabling problems," European Journal of Operational Research, vol. 219, no. 3, pp. 727-737, 2012.

[7] T. Huisman and R. J. Boucherie, "Running times on railway sections with heterogeneous train traffic," Transportation Research Part B: Methodological, vol. 35, no. 3, pp. 271-292, 2001.

[8] M. J. C. M. Vromans, R. Dekker, and L. G. Kroon, "Reliability and heterogeneity of railway services," European Journal of Operational Research, vol. 172, no. 2, pp. 647-665, 2006.

[9] R. M. P. Goverde, "Railway timetable stability analysis using max-plus system theory," Transportation Research Part B: Methodological, vol. 41, no. 2, pp. 179-201, 2007.

[10] Q. Y. Peng, J. J. Bao, C. Wen et al., "Evaluation theory and method of high-speed train diagrams," Journal of Southwest Jiaotong University, vol. 48, no. 6, pp. 969-974, 2013.

[11] T. Zhu, H. Wang, and B. Lu, "Method for optimally selecting the train diagram of passenger dedicated line based on Vague sets," China Railway Science, vol. 32, no. 4, pp. 122-127, 2011.

[12] W. L. Gau and D. J. Buehrer, "Vague sets," IEEE Transactions on Systems, Man and Cybernetics, vol. 23, no. 2, pp. 610-614, 1993.

[13] P. Vansteenwegen and D. Van Oudheusden, "Developing railway timetables which guarantee a better service," European Journal of Operational Research, vol. 173, no. 1, pp. 337-350, 2006.

[14] Y. Zhang, C. Tian, X. Jiang, and Y. Wang, "Calculation method for train headway of high speed railway," China Railway Science, vol. 34, no. 5, pp. 120-125, 2013.

[15] A. Charnes, W. W. Cooper, and E. Rhodes, "Measuring the efficiency of decision making units," European Journal of Operational Research, vol. 2, no. 6, pp. 429-444, 1978.

[16] W. W. Cooper, L. M. Seiford, and K. Tone, Data Envelopment Analysis, vol. 71, Kluwer Academic Publishers, New York, NY, USA, 2004. 


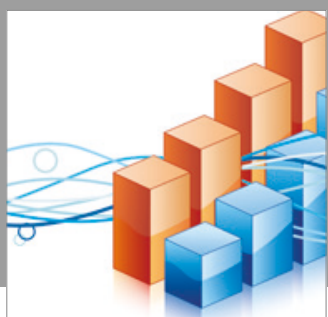

Advances in

Operations Research

vatersals

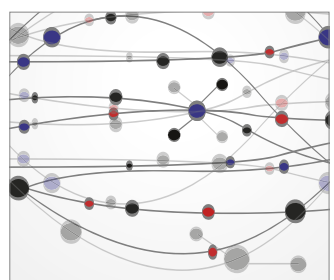

\section{The Scientific} World Journal
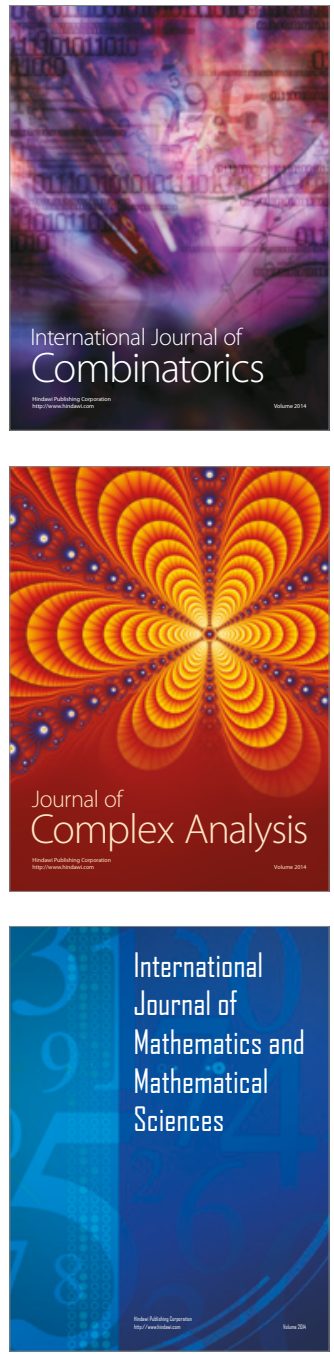
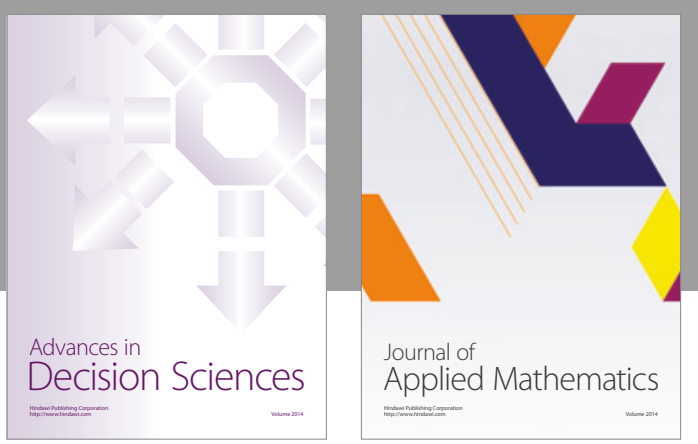

Algebra

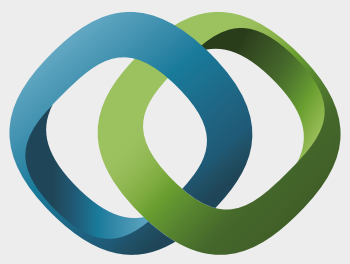

\section{Hindawi}

Submit your manuscripts at

https://www.hindawi.com
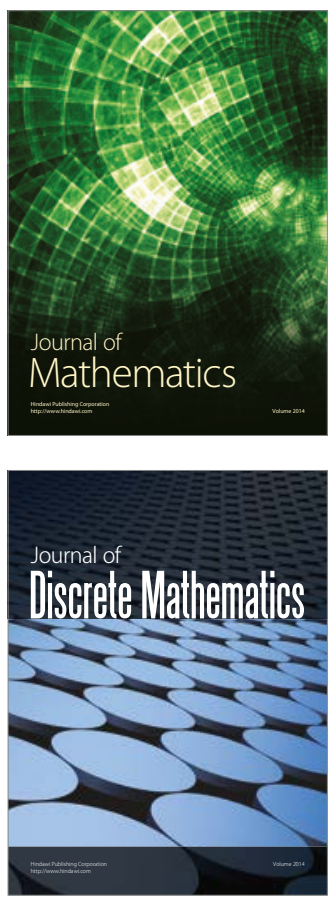

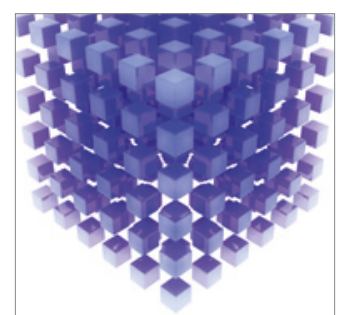

Mathematical Problems in Engineering
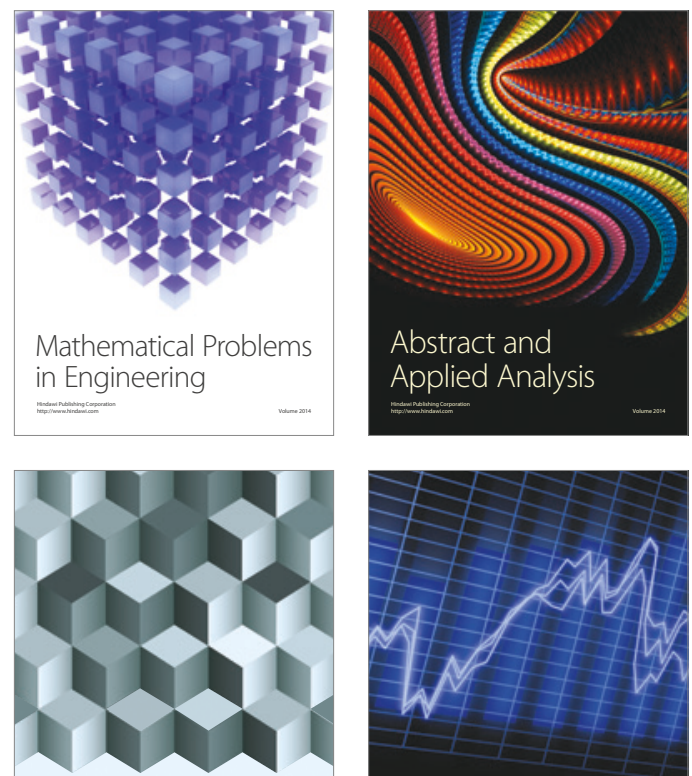

Journal of

Function Spaces

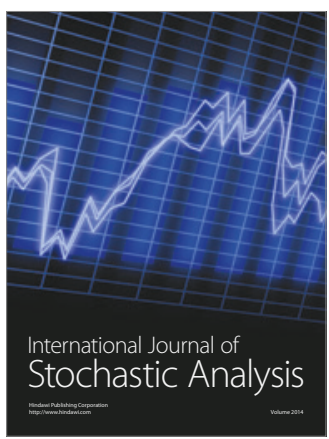

Probability and Statistics
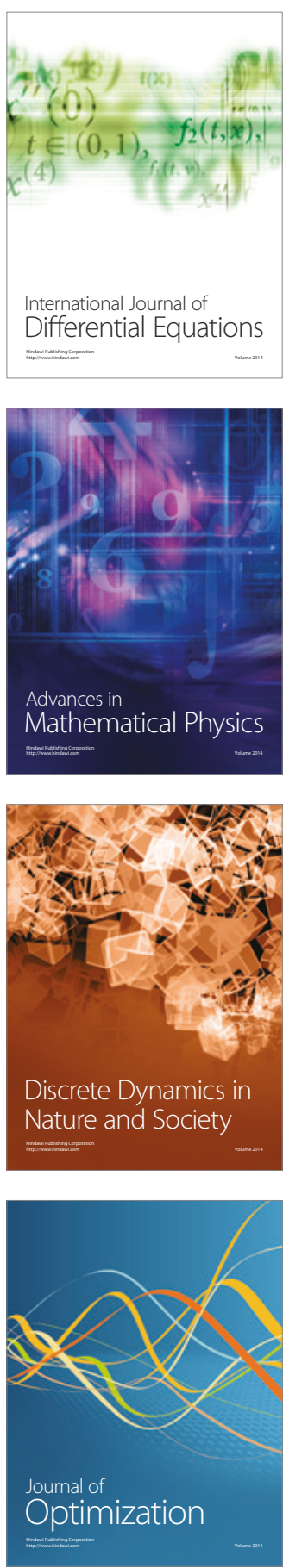